DOI: https://doi.org/10.22206/ceyn.2021.v5i1.pp71-96

\title{
LOGÍSTICA E INTERNACIONALIZACIÓN DE LAS EMPRESAS ANTES Y DURANTE LA PANDEMIA DEL COVID-19. BREVE REVISIÓN DE LITERATURA ESPECIALIZADA
}

\section{Ehyder Mario Barbosa Pérez ${ }^{a}$, Dustin Tahisin Gómez Rodríguez y Carlos Arturo Téllez Bedoya ${ }^{c}$}

\author{
Recibido: 18/11/2020 - Aprobado: 24/05/2020
}

Cómo citar: Barbosa Pérez, E. M., Gómez Rodríguez, D. T., \& Téllez Bedoya, C. A. (2021). Logística e internacionalización de las empresas antes y durante la pandemia del Covid-19. Breve revisión de literatura especializada. Ciencia, Economía y Negocios, 5(1), 71-96. https://doi.org/10.22206/ceyn.2021.v5i1.pp71-96

\section{Resumen}

El presente articulo tiene como objetivo general caracterizar las principales teorías y autores de la logística y la internacionalización antes de la pandemia, $y$ las estrategias que han utilizado en América Latina para tratar de contrarrestar los efectos del Covid 19 en las cadenas de valor. La metodología es de corte cualitativa y el método es de revisión documental. La principal conclusión es que la pandemia ha reflejado las asimetrías del comercio mundial. Donde se pueden identificar organizaciones que se adaptan en relación con su parque empresarial y buscan la manera de sortear la contingencia, o las que no pudieron con la misma. Asimismo, se ha puesto como pivote la resiliencia de los empresas dentro de las cadenas globales, ya que el mundo que viene, las organizaciones que se sumerjan en los cambios que exige la cadena global de suministros competirán y se quedarán o ampliarán su nicho de mercado.

Palabras clave: COVID 19; comercio; logística; internacionalización; organización.

Códigos JEL: F01, F19, L81, M16.

\footnotetext{
${ }^{a}$ Unidad Administrativa Especial de Organizaciones Solidarias. Bogotá, Colombia. ORCID: 0000-0001-5105-4496, Correo-e: ehyder.barbosa@orgsolidarias.gov.co

b Universitaria Agustiniana, Facultad de Ciencias Económicas y Administrativas. Bogotá, Colombia. ORCID: 0000-0001-5359-2300

Correo-e: dustin.gomez@uniagustiniana.edu.co;

c Universidad Jorge Tadeo Lozano. Facultad de Ciencias Económicas y Administrativas, Bogotá, Colombia. ORCID: 0000-0002-4568-0690

Correo-e: carlosarturo156@hotmail.com
} 


\title{
LOGISTICS AND INTERNATIONALIZATION OF COMPANIES BEFORE AND DURING THE COVID-19 PANDEMIC. A BRIEF REVIEW OF SPECIALIZED LITERATURE
}

\section{Ehyder Mario Barbosa Péreza , Dustin Tahisin Gómez Rodríguez and Carlos Arturo Téllez Bedoya ${ }^{c}$}

Received: 11/18/2020 Approved: 05/24/2021

\begin{abstract}
The general objective of this article is to characterize the main theories and authors of logistics and internationalization before the pandemic and the strategies they have used in Latin America to try to counteract the effects of Covid 19 in value chains. It is qualitative and the method is of documentary review. The main conclusion is that the pandemic has reflected the asymmetries in world trade. Where you can identify organizations that adapt in relation to your business park and look for a way to overcome the contingency or those that could not with it. As well as, the resilience of companies within global chains has been placed as a pivot, since in the world to come, organizations that immerse themselves in the changes required by the global supply chain will compete and stay or expand their market.
\end{abstract}

Keywords: COVID 19; commerce; logistics skills; internationalization; organization.

JEL codes: F01, F19, L81, M16.

\footnotetext{
${ }^{a}$ Unidad Administrativa Especial de Organizaciones Solidarias. Bogotá, Colombia. ORCID: 0000-0001-5105-4496, E-mail: ehyder.barbosa@orgsolidarias.gov.co

b Universitaria Agustiniana, Facultad de Ciencias Económicas y Administrativas. Bogotá, Colombia. ORCID: 0000-0001-5359-2300

E-mail: dustin.gomez@uniagustiniana.edu.co;

c Universidad Jorge Tadeo Lozano. Facultad de Ciencias Económicas y Administrativas, Bogotá, Colombia. ORCID: 0000-0002-4568-0690

E-mail: carlosarturo156@hotmail.com
} 


\section{Introducción}

Las transformaciones sociales como productivas de los agentes económicos desde la década de 1950 han reconfigurado la administración, la producción y la distribución de bienes y servicios tanto a nivel internacional como nacional. En efecto, el crecimiento económico de los países denominados del "primer mundo" entre las décadas de 1950 a 1970 fue vertiginoso. No obstante, el modelo económico de ese entonces se basaba en la política fiscal expansiva, la cual, a medida que pasó el tiempo, comenzó a generar variables negativas macroeconómicas como el aumento de la inflación y el acrecentamiento ineficiente de la burocracia, lo que, entre otras manifestaciones, condujo al agotamiento del modelo y la eclosión de nuevas formas de interpretar la realidad económica. Es así como, en la década de 1970, emergen críticas fuertes frente al modelo, las cuales desde la nueva escuela monetarista en cabeza de sus Premios Nobel de economía (Friedman, 1992; Hayeck, 2008), establecerán un formulario desde el discurso de la "libertad económica", sobre la manera como los agentes económicos deberán afrontar las sucesivas décadas, dado los cambios vertiginosos de la economía.

Precisamente, la nueva ideología que en el correr del tiempo se va a denominar neoliberalismo, enfatiza la necesidad de un modelo que desregule la actividad financiera, apoye la venta de los activos del Estado en el mercado, justifique los tratados de libre comercio, reivindique los postulados de la economía clásica y deslegitimice las "bondades" del Estado de bienestar que, según los autores neoliberales, ocasionaban pérdida adquisitiva de los consumidores como fenómenos inflacionarios, y que eran dinámicas propias de la política monetaria y no de la política fiscal. (Ferrero, 2002; 2007).

Al unísono, emerge el discurso de la globalización y, sobre todo, el de la deslocalización de los factores productivos como estrategia para ser más competitivo por parte de las empresas; toma relevancia la internacionalización de estas como la logística que utilizan en cada una de sus cadenas de abastecimiento. (Kalmanovitz, 2019; Comisión Económica para América Latina y el Caribe -CEPAL, 2018; 2019). No obstante, en 
el año 2020 surge una nueva pandemia que amplía y reta los desarrollos logísticos e internacionales de las empresas.

Por ello, el presente artículo tiene como objetivo general caracterizar las principales teorías y autores de la logística y la internacionalización antes de la pandemia, y las estrategias que han utilizado en América Latina para tratar de contrarrestar los efectos del Covid 19 en las cadenas de valor. Con el mismo propósito, la hipótesis central es que el año 2020 fue catastrófico para el volumen comercial de América Latina, en virtud de que en promedio las empresas no tenían un plan de emergencia como contrapeso frente a la pandemia. No obstante, en la medida que pasa el tiempo la resiliencia es más alta y esta se ha reflejado en estrategias que han tratado de responder a los tiempos de incertidumbre.

La metodología es de corte cualitativa y el método es una revisión de literatura especializada, que busca identificar las diferencias y convergencias de los autores a través de matrices que reflejan diferentes rubros (Gómez, Carranza \& Ramos 2017b; 2016): el marco teórico que utiliza el documento, el objetivo de este para entender la línea argumentativa, la metodología para saber el corte, etc. (Salcedo, 2015). De igual manera, en la primera parte se toman para el periodo de estudio documentos de la década de los 80, etapa en la que se inicia la ideología del neoliberalismo en América Latina (Bértola \& Ocampo, 2012), para la segunda, el año 2020.

Finalmente, el artículo se divide en varios apartados. En primer lugar, la introducción, donde se expuso de manera general el tema; luego, se presenta la primera caracterización de la internacionalización y la logística de las empresas antes de la pandemia, desde el punto de vista de sus autores, teorías y aportes. Como tercer acápite, se desarrolla la descripción de las estrategias que se utilizaron en América Latina para contrarrestar los impactos del Covid 19 en relación con la logística y la internacionalización. Por último, se formulan unas breves conclusiones.

\section{La internacionalización y la logística antes de la pandemia}

A continuación, en la tabla 1 , las diferentes teorías de internacionalización, autores y aportes. 


\section{Tabla 1. Teorías de la internacionalización de las empresas}

\begin{tabular}{|c|c|c|}
\hline $\begin{array}{l}\text { Teorías sobre el } \\
\text { proceso de Inter- } \\
\text { nacionalización } \\
\text { de las empresas }\end{array}$ & Autores & Aportes \\
\hline $\begin{array}{l}\text { Teorías de la Inter- } \\
\text { nacionalización }\end{array}$ & $\begin{array}{l}\text { (Liendo \& } \\
\text { Martínez, 2011) y } \\
\text { (Ballvé, 1992). }\end{array}$ & $\begin{array}{l}\text { Según los autores las variables que se deben de tener en } \\
\text { cuenta para de internacionalización de las PYME son: } \\
\text { 1. La asociación, que aborda primordialmente el problema } \\
\text { de competitividad por falta de escala. } 2 \text {. Objetivos comunes } \\
\text { entre los beneficiarios, procediendo con responsabilidad } \\
\text { frente a los posibles riesgos, pero sin disipar la independen- } \\
\text { cia de cada colaborador. }\end{array}$ \\
\hline $\begin{array}{l}\text { Teoría Eclética del } \\
\text { Comercio Interna- } \\
\text { cional }\end{array}$ & $\begin{array}{c}\text { (Dunning, 1981; } \\
\text { 1988a;1988b;1992) }\end{array}$ & $\begin{array}{l}\text { Su teoría expone una columna importante en el comercio } \\
\text { internacional, el cual denominó: "El Riesgo de Inversión", } \\
\text { el cual se desglosa en cuatro perspectivas: 1. La PYME } \\
\text { industrial o manufacturera. 2. La empresa de alta tecno- } \\
\text { logía "nacida global". 3. Las definiciones de rendimiento } \\
\text { internacional de las empresas asentadas en la teoría de los } \\
\text { recursos. 4. Las contribuciones de las teorías que explican el } \\
\text { fenómeno exportador desde una perspectiva de redes. }\end{array}$ \\
\hline \multirow{4}{*}{$\begin{array}{l}\text { Teorías de la Inter- } \\
\text { nacionalización } \\
\text { desde el enfoque } \\
\text { económico }\end{array}$} & (Root, 1994) & $\begin{array}{c}\text { El autor concibe la internacionalización como un conglo- } \\
\text { merado de operaciones que proporcionan las relaciones } \\
\text { entre las empresas y los mercados internacionales, por } \\
\text { medio de la proyección de estas. }\end{array}$ \\
\hline & (Hymer, 1960) & $\begin{array}{c}\text { Los procesos de internacionalización residen en el análisis } \\
\text { de la naturaleza y causas de la inversión extranjera (1960). } \\
\text { Una empresa debe aprovechar una ventaja competitiva } \\
\text { exclusiva, que posee en el mercado externo, aún si esta no } \\
\text { es evidente (1976). }\end{array}$ \\
\hline & $\begin{array}{c}\text { (Buckley \& Casson, } \\
1976)\end{array}$ & $\begin{array}{l}\text { La internacionalización de las empresas se asienta por dos } \\
\text { variables: 1. Que coexistan ventajas de instaurarse en el } \\
\text { exterior 2. Que sea viable la internacionalización a cambio } \\
\text { de venderlas o cederlas. En consecuencia, según los autores, } \\
\text { estas variables fijaran si la empresa se debe externalizar o } \\
\text { mejorará los procesos internos de la misma. }\end{array}$ \\
\hline & $\begin{array}{l}\text { (Vernon, 1966) y } \\
\text { (Kojima, 1982) }\end{array}$ & $\begin{array}{l}\text { La internacionalización se logra por medio de ostentar } \\
\text { ventaja comparativa de un producto fuerte en producción } \\
\text { y exportación, conforme a su posición geográfica. De igual } \\
\text { manera plantea una teoría macroeconómica en la cual ma- } \\
\text { nifiesta que las ventajas comparativas que tienen los países } \\
\text { deben ser aprovechadas por aquellos que no la tienen. }\end{array}$ \\
\hline \multirow[b]{2}{*}{$\begin{array}{l}\text { Teoría de la Inter- } \\
\text { nacionalización } \\
\text { por procesos }\end{array}$} & (Ellis, 2000) & $\begin{array}{c}\text { Enseña que el ingreso a los mercados externos es avistado } \\
\text { como función de las interacciones inter organizativas paula- } \\
\text { tinas de las empresas locales y sus redes. }\end{array}$ \\
\hline & $\begin{array}{l}\text { (Blankenburg \& } \\
\text { Eriksson, 2000); } \\
\text { (Blankenburg \& } \\
\text { Johanson, } 1992\end{array}$ & $\begin{array}{c}\text { Los autores especifican que las coyunturas de iniciación a } \\
\text { los mercados externos por parte de la empresa local son por } \\
\text { medio los elementos que conforman una red de empresas } \\
\text { propias en el exterior, es decir, (multinacionales), o en coa- } \\
\text { liciones con empresas extranjeras (Join Ventures o Alianzas } \\
\text { Internacionales). (Blankenburg, Eriksson, Johanson, 1999; } \\
\text { Ford, 1980). }\end{array}$ \\
\hline
\end{tabular}

\section{Fuente: elaboración propia.}


En las tablas 2 y 3 , se presentan los principales autores consultados, las líneas argumentativas de sus teorías y el nombre del modelo de logística.

Tabla 2. Modelos de logística

\begin{tabular}{|c|c|c|}
\hline Autores & Teoría & Modelo \\
\hline $\begin{array}{l}\text { (Harrington, 1996; } \\
\text { 1998; 1999) }\end{array}$ & $\begin{array}{l}\text { Se fundamenta en cambiar la perspectiva } \\
\text { centrada en la organización, sino en los } \\
\text { procesos y se apoya en cinco pasos. }\end{array}$ & $\begin{array}{l}\text { Modelo de Mejora- } \\
\text { miento de los Procesos } \\
\text { de la Empresa }\end{array}$ \\
\hline $\begin{array}{l}\text { (Acevedo \& Gómez, } \\
\text { 1996) } \\
\text { (Acevedo, Urquiaga \& } \\
\text { Gómez, 1997; 1996) }\end{array}$ & $\begin{array}{l}\text { Modela el funcionamiento del sistema de } \\
\text { producción, teniendo en cuenta variables } \\
\text { conectadas que constituyen las medidas } \\
\text { de su actividad, valorando las incidencias } \\
\text { que conducen al perfeccionamiento. }\end{array}$ & $\begin{array}{c}\text { Modelo de Evaluación } \\
\text { del Sistema de Produc- } \\
\text { ción }\end{array}$ \\
\hline (Forrester, 1958; 1961) & $\begin{array}{l}\text { Los flujos de dinero, las órdenes, los } \\
\text { materiales, el recurso humano y el equipa- } \\
\text { miento como variables del flujo de infor- } \\
\text { mación. Donde este último se concibe } \\
\text { como una división de etapas del trabajo } \\
\text { para obtener resultados. }\end{array}$ & $\begin{array}{l}\text { Modelo de Dinámica } \\
\text { Industrial }\end{array}$ \\
\hline (Long, 2005) & $\begin{array}{l}\text { La logística internacional se puede } \\
\text { entender desde tres enfoques: el enfoque } \\
\text { teórico: que comprende la logística, desde } \\
\text { sus fundamentos, su historia, su entorno y } \\
\text { su relación con las economías globales. El } \\
\text { enfoque administrativo: se basa en deci- } \\
\text { siones estratégicas. } \\
\text { El enfoque práctico y técnico: su percep- } \\
\text { tiva es desde lo operativo, donde se exte- } \\
\text { riorizan las características propias de la } \\
\text { logística en su cotidianidad }\end{array}$ & $\begin{array}{l}\text { Modelos Basados en } \\
\text { el conocimiento // } \\
\text { Administración de la } \\
\text { cadena de abasteci- } \\
\text { miento global }\end{array}$ \\
\hline (Ballou, 2004) & $\begin{array}{l}\text { Este modelo va en sintonía con la logís- } \\
\text { tica y la cadena de suministro, en virtud } \\
\text { de que este segmento la administración } \\
\text { como la planeación estratégica es esencial } \\
\text { para la competitividad. }\end{array}$ & $\begin{array}{l}\text { Modelos Basados en el } \\
\text { conocimiento. }\end{array}$ \\
\hline
\end{tabular}

Fuente: elaboración propia 
Tabla 3. Modelos de logística

\begin{tabular}{|c|c|c|}
\hline Autores & Teoría & Modelo \\
\hline $\begin{array}{c}\text { Freeman } \\
(1984)\end{array}$ & $\begin{array}{l}\text { Teoría de los stakeholders // Gestión } \\
\text { Estratégica: un enfoque de las } \\
\text { partes interesadas, hace hincapié } \\
\text { en la importancia de invertir en } \\
\text { las relaciones con los que tienen } \\
\text { una participación en la empresa. } \\
\text { La estabilidad de estas relaciones } \\
\text { depende del intercambio de, al } \\
\text { menos, un núcleo de principios } \\
\text { o valores. Por lo tanto, la teoría } \\
\text { de las partes interesadas permite } \\
\text { incorporar los valores personales en la } \\
\text { formulación y ejecución de los planes } \\
\text { estratégicos. }\end{array}$ & $\begin{array}{c}\text { Modelos Basados en el } \\
\text { Conocimiento//Teoría de } \\
\text { los stakeholders }\end{array}$ \\
\hline $\begin{array}{c}\text { (Russo \& } \\
\text { Fouts, 1997) }\end{array}$ & $\begin{array}{l}\text { Desde la teoría de los recursos de } \\
\text { la empresa, los autores conciben, } \\
\text { que tanto el medio ambiental } \\
\text { como el impulso económico están } \\
\text { correlacionados. }\end{array}$ & $\begin{array}{c}\text { Modelos Basados en el } \\
\text { Conocimiento//Teoría de } \\
\text { Recursos }\end{array}$ \\
\hline
\end{tabular}

Fuente: elaboración propia.

\subsection{Internacionalización}

Los fenómenos de la globalización de los mercados son procesos en los cuales no pueden escapar ni las organizaciones ni los consumidores (Rueda, 2008). En efecto, estos fenómenos estimulan la idea tentadora de ampliar el número de compañías que comercialicen sus productos en el exterior, convirtiéndose en empresas cada vez más productivas y competitivas, que favorezcan el beneficio económico y social de una nación. No obstante, es necesario reconocer que internacionalizar una organización no es una tarea fácil, sino que, por el contrario, puede llegar a ser riesgoso y complejo; este tipo de esfuerzos suelen ser altamente premiados, principalmente cuando las organizaciones se focalizan primero en la indagación de información y conocimientos previos de las necesidades y expectativas de los compradores en los nuevos mercados meta. 
Precisamente, sostiene Villarreal (2005), que equiparar la heterogeneidad intrínseca o procurar una delimitación conceptual de la internacionalización de las empresas como una posible estrategia de una empresa multinacional como entidad organizativa es reducirla. Por ende, esta gestión debe de ser extensa, ya que con ello se tiene una consideración multidimensional del concepto. De ahí que la identificación de las ventajas de localización en mercados exteriores en función de factores económicos: costes, economías de escala, estructura del mercado son procesos graduales de toma de decisiones acumulativas, basadas en las interacciones permanentes entre el desarrollo del conocimiento sobre mercados exteriores y el compromiso de recursos en dichos mercados (Armario, Rastrollo \& González, 2008).

En el mismo sentido, la internacionalización de las empresas es el gran reto del presente, ya que el mercado interior no favorece en el desarrollo más allá de un tercio de estas, de cara al futuro. En consecuencia, el nuevo espacio económico se definirá por una mayor competitividad, constituida en factores tan complejos como la calidad, el diseńo, la innovación, la capacidad de adaptación a los contextos y a las coyunturas. Por ello, si se pretende ser competitivo desde el ámbito internacional es imperativo comprender la configuración de las grandes áreas comerciales, tales como la Comunidad Europea, USA, Canadá y Este asiático, sin olvidar el complejo mercado del Este europeo (Ballvé,1992).

Por otra parte, Larraya (1994) dimensiona variables negativas en el proceso de la internacionalización de las empresas. Como son la minúscula anchura empresarial, la exigua cooperación interempresarial, la escasa planificación de la actividad exterior, así como la mínima dotación de recursos para dicha actividad. Sin olvidar la imperiosa necesidad de una mayor alineación profesional hacia los mercados exteriores entre los recursos humanos (directivas y empleados), la precaria innovación en el producto final, la limitada cartera de clientes, el trato alejado y notablemente mejorable a clientes, la exigua estabilidad en los volúmenes de exportación, las carencias en la estructura financiera que limitan la financiación de las operaciones en el exterior, asimismo, la mínima utilización de los apoyos institucionales en el exterior. 
Por consiguiente, una de las formas en las que se puede mejorar lo antes dicho son las explicaciones realizadas por Sáez y Melo (2002), quienes analizan el apoyo financiero que facilitó el Banco de Desarrollo de Brasil (BNDES), el cual, a juicio de los autores, se convirtió en un punto clave para el proceso de internacionalización de las empresas brasileńas, en virtud de que colaboró tanto en el desarrollo de infraestructura de nuevas tecnologías como de procesos sistematizados para la creación de productos. De igual manera, para que una empresa pueda variar, es ineludible generar una serie de escenarios, que conjuntamente y como un todo, formen la estructura de las cuatro áreas de la organización para su proyección en el mercado internacional. Por lo tanto, puede que la organización tenga más adelantadas algunas etapas en unas áreas más que en otras. Por ello, antes de tratar de moverse hacia una etapa superior, la empresa debe estandarizar todas aquellas que están en un nivel inferior al nivel en donde se encuentran las de mejor posición.

Ahora bien, "la internacionalización se puede entender de igual manera como una serie de modelos" (Castro, 2009, p. 41) donde su fin es sugerirles a las empresas de poder participar en los mercados internos y externos para maximizar sus ingresos económicos. Desde esta perspectiva, los componentes para la internacionalización de las pequeña y mediana empresa (PYME) en un caso específico como es la sociedad colombiana son los siguientes: la asociatividad, globalización-apertura, apoyo y desarrollo empresarial, TIC, financiación y costos, gerencia y visión internacional, gobernabilidad, ciudad región-infraestructura, característica, producto y/o servicio, medio ambiente y sostenibilidad, mercado y logística y riesgo comercial y político-riesgo país, siendo estos los descritos por Urrea y Abello (2011). Precisamente, las estrategias para mejorar la competitividad de las empresas colombianas en su proceso de internacionalización pueden ser las siguientes: transformación productiva, atracción de inversión, turismo de clase; las cuales fueron establecidas en el Plan Estratégico Exportador 1999 - 2009. Un punto más para la mejora de la competitividad en las Pyme colombianas también es la asociatividad por las unidades empresariales, siendo esto la base para la Fundación de Estudios Sociológicos (FUNDES) y Exportaciones 
Turismo Inversión Marca País (Procolombia) en el año 2004, en su creación del Programa de Redes Empresariales para Internacionalización en el marco del mejoramiento de la competitividad de las PYME colombianas (Correa, Durán \& Segura, 2010).

Por otra parte, la internacionalización desde una perspectiva de desarrollo local, presenta diversos horizontes (Osorio, 2010), siendo estos los procesos necesarios para la internacionalización de las microempresas colombianas; un enfoque correspondiente a la dirección teórica de las fases, del proceso como del ciclo de vida de las empresas que se internacionalizan.

Ahora bien, enfatizando en el caso colombiano, Urrea y Abello (2011) afirman, que el principal foco de apoyo es el sector manufacturero, en virtud de que el país recibe el mayor rubro de ingresos por medio de sus exportaciones. Sin embargo, algo que jalonó este proceso, dado el impacto de la Pyme en la sociedad colombiana, fue la creación del Plan Nacional para el Desarrollo de la Microempresa, que impulsó según el crecimiento de estas.

Otro ejemplo es Interconexión Eléctrica S.A (ISA), cuya estrategia es pasar a la historia como la compañía que desarrolla los proyectos de infraestructura de Colombia. ISA evoluciona, avanza, amplía su horizonte, diversifica sus capacidades, pero conserva algo inalterable: su compromiso con el desarrollo de los países, las regiones y las personas. Una empresa como ISA, les muestra a sus accionistas que consolida su estrategia de crecimiento y que continúa generando valor para sus diferentes grupos de interés (Escobar \& Chaparro, 2011). En el mismo sentido, para Federación Nacional de Cuero - Fedecueros (2013) con las condiciones negativas en que se encuentra Colombia, con respecto a otros países de la región, genera planes de negocio que buscan que un país como Colombia se convierta en una potencia mundial en la elaboración de manufacturas de cuero, diseńando un plan ambicioso al año 2028, donde se propone la creación de cooperativas de trabajo, tecnificación de este sector, y un incremento en la calidad de las materias primas. Decisivamente el gobierno colombiano debe enfocarse al mismo tiempo en tres áreas si se quiere ser competitivo en un mundo que tiende a ser globalizado:

La búsqueda de un mayor acceso a más mercados, (2) la eliminación del sesgo anti exportador y (3) la superación de obstáculos básicos a 
la competitividad de la economía colombiana. De lo contrario[,] el progreso de tipo exploratorio descriptivo de que se logre en una de ellas se verá frustrado por el atraso de las otras dos y no se conseguirá una mayor inserción global de la economía (Reina, 2010, p. 4).

\subsection{Logística}

Con el mismo propósito, los procesos logísticos de las empresas han tenido también que adaptarse a las nuevas exigencias del mercado. En virtud de que la economía mundial tiende a ser globalizada y con problemas fuertes de contaminación, donde los clientes cada vez son más exigentes. De ahí que las empresas conciban la internacionalización y la competitividad, ejes trasversales de sus estrategias comerciales. De donde se sigue que los procesos logísticos según la literatura especializada son un dinamizador del proceso (Díaz, Pizza \& Salamanca, 2013).

Precisamente en la tabla 2 del presente capítulo se pueden identificar los diferentes modelos que se utilizan en promedio desde el punto de vista logístico. Entre ellos, el modelo de investigación analítico (Ballesteros, Ballesteros y Duarte, 2009), el cual concluye que la innovación, la tecnología y las alianzas son tendencias que concuerdan con los postulados del desarrollo sostenible, lo cual genera una mejor calidad de vida, como la tan anhelada competitividad desde la logística, y la necesidad de que los procesos logísticos implementen políticas y normas que ayuden a planificar, controlar y ajustar cada una de las etapas.

De igual manera, al analizar la literatura referente a la Logística Inversa, se concibe la misma como una estrategia para que las cadenas de suministro y empresas preserven el medio ambiente y agencien convenientemente las devoluciones (Gómez, 2006). El objetivo de ello es optimizar y redimir valor a los productos, por medio del reciclaje a nivel nacional como internacional.

Con el mismo propósito, Peña, Torres, Vidal y Marrnolejo (2013) realizan una reflexión sobre la logística inversa y la integral, como la Gestión Integral de Residuos Sólidos (GISRS) en el aparato productivo. Su conclusión más relevante es que dicha logística promueve impactos reducidos para este sector productivo de Colombia y que es imperativo 
generar decisiones de tipo gubernamental y empresarial para mejorar tanto los beneficios económicos como los ambientales. En igual sentido, desde el punto de vista internacional, se ha evidenciado que donde lo jurídico es fuerte frente a la contaminación de las empresas, como es el caso del norte de Europa, se desarrollan ventajas competitivas. De ahí que la utilización sistemática de la producción limpia como la logística inversa y el ecodiseńo de productos desarrolle esta capacidad, tal como se ha identificado en países líderes como Alemania y en algunas empresas españolas (Tamayo, Azucena \& Molina, 2007).

De todos modos, la competitividad desde el punto de vista logístico y su gestión de la cadena de suministros, tiene que ver con las creencias medioambientales de los directivos, con la concienciación, el tamaño empresarial, como se expuso anteriormente, y las certificaciones que tenga las empresas desde el punto de vista ambiental, sin olvidar la presión de los stakeholders, los grupos internacionales y su dislocación de la producción, y del sector en el cual se encuentra inmersa la empresa. Es decir, sus competidores. De ahí que la articulación de ellas son determinantes para mejorar la cadena de valor y el compromiso frente al medioambiente de la función logística (González \& González, 2008).

En contraste, la logística integral, la hiperconectividad dentro de un contexto global de las empresas pequeñas y medianas son variables de competitividad. Un caso en el cual se podría rediseñar y mejorar teniendo prácticas eficientes frente al medio ambiente es en el sector del calzado, donde se puede consolidar para la creación de valor agregado al sector (Villarreal, 2012). Otro caso puede ser el Estado español, que está consciente de que sus empresas, tanto las medianas como las pequeńas, tienden a operar desarticuladas, además de que poseen precarios resultados desde el orden de la eficiencia y la eficacia de sus procesos. Por ello, una forma de mejorar estas falencias es desarrollando modelos que optimicen los procesos logísticos, específicamente en los Distritos Internacional de Agronegocios - DIA, el cual integra los procesos de planeación por medio del modelo Supply Chain Operations Reference Model (SCOR), utilizando la norma ISO 90001:2000 la cual puede ser una manera para rediseñar y diagnosticar los procesos de las empresas, dando como resultado un diferenciador, del cual pueden ser más competitivas (Arellano Ríos, Carballo \& Flérida, 2009). Decisivamente las pequeñas y medianas empresas colom- 
bianas podría utilizar estos modelos para insertarse en la economía global como vehículo de competitividad (Castro, 2009; Porter, 1985).

\section{Descripción de la internacionalización y la logística de las empresas durante la pandemia en América Latina}

\subsection{Características del comercio en la pandemia de América Latina}

En un mundo que tiende a ser globalizado, los cambios, las irrupciones pueden repercutir en la gestión y organización de las empresas y más cuando es una pandemia. Precisamente, se podría afirmar que la gran mayoría de las empresas han sufrido choques por el COVID-19, reflejando las grandes asimetrías en las cadenas globales de valor frente a esta contingencia (Fransoo \& Udenio,2020; Kalish, 2020). De ahí, que varios países y para el presente artículo delimitado en América Latina hayan iniciado estrategias productivas y comerciales, con el objetivo de reducir la interdependencia con otras economías.

En efecto, el sector logístico no ha sido la excepción. Las organizaciones han tenido que mejorar y desarrollar dos requisitos. Por un lado, certificar los envíos al tiempo contratado y, por el otro, aplicar la bioseguridad (Velasco y Gómez, 2020). Lo cual ha contribuido al acrecentamiento de los costes derivados de adecuar lo antes mencionado (Enfoka, 2020; Rivera, 2020).

En particular, a la llegada del COVID 19 se le suma el debilitamiento del comercio exterior mundial, sobre todo después de las crisis de 2008. Mientras que en los periodos 1990-2007 el volumen de comercio creció en un 6,2\%, en los años 2012 al 2019 se redujo en un 2,3\% anual. Con la coyuntura del COVID 19, a mayo de 2020, este cayó en un 17,7 \% en relación con el 2019. Desde el punto de vista de América Latina, tanto las exportaciones como las importaciones en promedio se redujeron en un 17 $\%$ entre los meses de enero y mayo, en relación con el año 2019. De igual manera, entre los meses abril - mayo en comparación con los mismos meses en el año $2009^{a}$ se redujeron $20 \%$ más las exportaciones y $25 \%$ más las importaciones (Transport Intelligence, 2020; CEPAL, 2020a).

\footnotetext{
a En este año se pudo apreciar mucho mejor los impactos de la crisis financiera del 2008
} (Stiglitz, 2012). 
Desde el punto de vista de costos de envíos para la región, este decreció entre enero y mayo a un $24 \%$. Donde el sector automotor toma la delantera con una caída cercana del $55 \%$, seguido de textiles, confesiones, calzado. Asimismo, respecto al comercio marítimo de América Latina, su variación anual en el periodo enero - marzo fue de -6,1 \% y se redujo aún más en abril - mayo. Sin embargo, los fletes aumentaron los precios si se compara con el año 2019, y al 2 de julio lo superaban con más del $45 \%$. Acotando, que la actividad portuaria de la región en contendores se redujo en un $1 \%$ y la suma de exportaciones e importaciones se contrajo en $6 \%$ (Leporati, Morales \& Martul, 2020; CEPAL, 2020b).

\subsection{Estrategias utilizadas en América Latina para contrarrestar la pandemia desde la logística y la internacionalización}

A continuación, se presentan un conjunto de estrategias que se pudieron identificar en la literatura especializada, consultada a través de la metodología y el método propuesto, tomando como base el año 2020:

A. Generación de redes de puntos de entrega y recogida de bienes y servicios. Al crearse estas redes se mejora la eficiencia de los procesos de distribución y trasporte, ya que reducen tanto los costes de entrega como de los posibles fallos por no estar el consumidor final al recibir (Fraso, 2020).

B. Creación de almacenes urbanos que mejoran la eficiencia de los procesos logísticos. Dada la limitación de la venta del parque automotriz, la distribución por medio de estos almacenes se ha consolidado como la estrategia más eficaz en términos medioambientales.

C. Utilización de aplicaciones para la optimización de las rutas de distribución. Precisamente, se ha podido evidenciar que el saber con exactitud dónde queda el lugar del destinatario se pueden reducir los costes de combustible, el tiempo de entrega y sobre todo reducir el packaging (Transport Intelligence, 2020).

D. Aumento del consumo local. Al estar confinados, hubo un regreso al consumo local (Sagè Gavin et al., 2020). Un ejemplo de ello es lo denominado como Circuitos Cortos de Comercialización- C.C.C. (Rendón \& Gómez, 2020). En términos generales, los C.C.C. son 
estrategias avaladas por la Food Agriculture Organization- FAO, que busca reducir la intermediación entre productor y consumidor final. Así mismo, tienen como objetivo, mejorar la calidad de vida de los campesinos, en virtud de que este al participar en esta estrategia recibiría más ingresos, se relacionaría con el comprador y sobre todo comercializa los productos frescos de la región en beneficio de su tejido social y empresarial (Barbosa, Insuasti \& Gómez, 2020; Barbosa \& Gómez, 2021).

E. La evolución de los clientes como sus gustos han cambiado. Por consiguiente, otra estrategia utilizada por algunas empresas de América Latina fue la utilización de las Tic y de procesos productivos más amigables con el medio ambiente. En términos económicos sostenibilidad débil. (Gómez, 2021; Enfoka, 2020).

F. Otra estrategias que desarrollaron algunas empresas de América Latina también tienen que ver con tres líneas: la adaptabilidad, como se ha mencionado; la relación oferta y demanda; y, por último, el transporte y almacenamiento en el lugar de trabajo. Decisivamente varias empresas confeccionaron mapas y redes con sus proveedores en relación con la fabricación y la distribución que ofrezca valor añadido. Asimismo, se han evaluado el estado y la disposición de los proveedores en relación con la continuidad de la empresa y las alternativas frente a la pandemia. De la misma forma, se refleja una transparencia en el verdadero coste de prestar el servicio, ya que esto ha contribuido en conocer los factores del coste, la demanda de los clientes, el nivel de servicio etc. En igual sentido, se ha revaluado los supuestos, en virtud de que los anteriores a la pandemia no son necesariamente los del presente. Sin olvidar, que la utilización del teletrabajo es a corto plazo no a largo, y abrir la posibilidad de capital humano con diferentes habilidades, dado que la contingencia cambió la logística y la internacionalización de las empresas (Wilding, Dohmarn \& Wheatkey, 2020; Stiglitz, 2020).

G. En términos generales, se puede identificar que, dependiendo del sector de la empresa, el grado de competitividad y la relación con el intercambio de capital tangible y no tangible generaron o están generando en América Latina una serie de estrategias. En particular, el grueso de ellas, se desprenden planes de emergencias donde se iden- 
tifican las características de la demanda, la localización de recursos, la adquisición de suministros, la logística de cada organización y la asignación, que en muchas fue una modelo de cooperación eficiente tendiendo como emblema el principio de asignación (DHL, 2020; Zandi, 2020).

H. Otra estrategia que tienen que ver con las redes es la propiciada por las alianzas pública privadas y las de colaboración de gobiernos. Ya que han potencializado la transparencia de las cadenas de suministro por medio de las TIC desde los datos compartidos como la recopilación de estos. Para terminar con la estrategia que se ha mejorado como lo es la infraestructura logística, que hace hincapié en las existencias nacionales. Esta última, desde la perspectiva de la predeterminación de servicios logísticos, las modalidades de trasporte como las rutas de suministro (Linton \& Vakil, 2020).

\section{Conclusiones}

El COVID 19 ha generado tanto cambios sustantivos como incertidumbre a nivel global. El paro disruptivo de la demanda de consumo de bienes y servicios ha impactado negativamente en los circuitos comerciales mundiales, donde las cadenas han sido golpeadas de manera contundente. Se han propagado los bloqueos, las interrupciones de vuelos, cierre de plantas industriales, limitación de cadenas de suministro en todo el mundo, ocasionado rupturas en las cadenas de abastecimiento. América Latina es una de las regiones más golpeadas tanto desde el punto de vista social como del aparato productivo, ya sea por la precaria competitividad de la gran mayoría de ecosistema empresarial, por la poca o nula colaboración con sus Estados o por la apremiante corrupción.

La pandemia ha reflejado las asimetrías del comercio mundial. Donde se puede apreciar las organizaciones que se adaptan en relación con su parque empresarial y buscan la manera de sortear la contingencia o las que no pudieron con la misma. Asimismo, se ha puesto como pivote la capacidad y resiliencia de los empresas dentro de las cadenas globales, dado que el mundo que viene, las organizaciones que se sumerjan en los cambios que exige la cadena global de suministros se mantendrán y se quedarán con el nicho de mercado. Un caso particular serán las 
empresas de América Latina, que tienen mucho que demostrar más allá de la economía extractiva.

Entre los desafíos para mejorar la competitividad de cada uno de los actores identificados en el presente artículo, desde la perspectiva de la logística como eje dinamizados tanto del sector importador como del exportador de bienes y servicios, y a la internacionalización como eje trasversal del discurso de la globalización, se encuentra el cambio incesante de capital tangible e intangible, en un mundo que tiene como objetivo la libertad económica pregonada por el discurso hegemónico del neoliberalismo. En efecto, los procesos logísticos como la internacionalización de las empresas son variables de incursión del comercio exterior. De ahí, que las nuevas dinámicas de la economía internacional hagan hincapié en tener como baluartes estas características, para poder mantenerse y expandirse en el comercio, en aras de continuar en el mercado.

Para terminar, el presente artículo es derivado del proyecto de investigación intitulado: Macroproyecto: Análisis, desarrollo, fomento y gestión del talento humano en el municipio de Aracataca, Magdalena desde la economía solidaria (2020-2022) en su segunda fase entre la Unidad Administrativa Especial de Organizaciones Solidarias - UAEOS y la Universidad Agustiniana, Bogotá, Colombia. En efecto, en el año 2021 se pretende efectuar circuitos cortos de comercialización como estrategia frente a la pandemia y los precarios índices socioeconómicos del territorio, y es necesario entender las características de la logística y la internacionalización. De ahí que para otro documento se esté gestionando ya los resultados y analizándolos desde una metodología cuantitativa. No obstante, la línea argumentativa del presente escrito está por encima del artículo y será para otra entrega.

\section{Referencias}

Acevedo, J. y Gómez, M. (1996). Sistemas logísticos. La Habana, Cuba: Ediciones Díaz De Santos.

Acevedo, J., Urquiaga, A. y Gómez, M. (1997). Rediseño de los sistemas logísticos para competir con éxito. La Habana, Cuba: Ediciones Díaz De Santos.

Acevedo, J., Urquiaga J. y Gómez, M. (1996). El modelo general de la organización, herramienta para el análisis de los sistemas logísticos. La Habana, Cuba: Ediciones Díaz De Santos. 
Arellano, A., Ríos, N., Carballo, B. y Flérida, F. (2009). Arquitectura de procesos de la cadena logística para empresas a integrarse al distrito internacional de agronegocios pyme. Scientia et Technica, 3(43), 141-146.

Armario, J., Rastrollo, M. y González, E. (2008). La internacionalización de la empresa: el conocimiento experimental como determinante del resultado en mercados exteriores.

Ballesteros, D., Ballesteros, P. \& Duarte, T. (2009). Contribuciones de la logística al desarrollo sostenible. Scientia et Technica, 15(41), 171-174. Doi: http://dx.doi.org/10.22517/23447214.2877

Ballou, R. (2004). Logistica. Administración de la cadena de suministro. México D.F. México: Pearson Educación.

Ballvé, P. (1992). La internacionalización de la empresa. Revista de estudios Agro-sociales. (161), 323 - 337. Recuperado de https:/www.mapa. gob.es/ministerio/pags/biblioteca/revistas/pdf_reas/r161_13.pdf

Barbosa, E., Insuasti, E., y Gómez, D. (2020). Las compras públicas y los circuitos cortos de comercialización como estrategias para la reactivación económica de las zonas rurales afectadas por COVID 19. Ponencia. III Congreso CATATUMBARÏ. Universidad Francisco de Paula Santander -Ocaña. Colombia.

Barbosa. E., \& Gómez, D. (2021). Economía solidaria y construcción de paz. Revista sur. Recuperado de: https://www.sur.org.co/economia-solidaria-y-construccion-de-paz/

Bértola, L., y Ocampo, J. (2012). El desarrollo económico de América Latina desde la independencia. FCE, México.

Blankenburg, D. \& Eriksson, K. (2000). The character of bridgehead relationships. International Business Review, 1(2), 91-210. Doi: https:// doi.org/10.1016/S0969-5931 (99)00036-0

Blankenburg, D. \& Johanson, J. (1992). Managing network connections in international business. Scandinavian Business Review, 1(1), 5-19. Doi: https://doi.org/10.1016/0962-9262(92)90033-3

Blankenburg, D., Eriksson, K. \& Johanson, J. (1996). Business networks in international business relationships. Journal of International Business Studies, 27(5), 1033-1055. Doi: https://doi. org/10.1057/9781137508829_6 
Blankenburg, D., Eriksson, K. \& Johanson, J. (1999). Creating value through mutual commintment to business network relationships. Strategic Management Journal, 20(4), 67-480. Doi: https://doi.org/10.1002/ (SICI) 1097-0266(199905)20:5<467::AID-SMJ38>3.0.CO;2-J

Buckley, A. \& Casson, M. (1976). The future of the multinational enterprise. Londres, Inglaterra: Macmillan.

Castro, A. (2009). Aproximación al proceso de Internacionalización de las empresas; El Caso Colombia. Facultad de Administración. Bogotá, Colombia: Editorial Universidad del Rosario.

Choon, K. (2001). A framework of supply chain management literature. European Journal of Purchasing \& Supply Management, 7(1) 39-48. Doi: https://doi.org/10.1016/S0969-7012(00)00020-4

Comisión Económica para América Latina y el Caribe (CEPAL). (2018). Comisión Económica para América Latina y el Caribe. Explorando nuevos espacios de cooperación entre América Latina y el Caribe y China. Santiago de Chile, Naciones Unidas, 2018.

Comisión Económica para América Latina y el Caribe (CEPAL) (2019). Comisión Económica para América Latina y el Caribe. Panorama Social de América Latina 2019. CEPAL. Recuperado en: https:// www.cepal.org/es/publicaciones/44969-panorama-social-america-latina-2019

Comisión Económica para América Latina y el Caribe (CEPAL). (2020a). Los efectos del COVID-19 en el comercio internacional y la logistica. CEPAL. Recuperado en: https://www.cepal.org/es/publicaciones/ 45877-efectos-covid-19-comercio-internacional-la-logistica

Comisión Económica para América Latina y el Caribe (CEPAL). (2020b). Comisión Económica para América Latina y el Caribe. Logística internacional pospandemia: Análisis de la industria aérea y la de transporte maritimo de contenedores. CEPAL. Recuperado en: https:// www.cepal.org/es/notas/logistica-internacional-pospandemia-analisis-la-industria-aerea-la-transporte-maritimo

Consejo Privado Competitividad. (2011-2012). Consejo privado de competitividad. Del Informe nacional de Competitividad. Recuperado de http://www.compite.com.co/site/wp-content/uploads/2011/11/ Resumen-ejecutivo.pdf. 
Correa, L., Durán, Y. y Segura, O. (2010). Redes empresariales para la internacionalización. Series Neustria Experience, (5), 9-44. Recuperado de http://www.eafit.edu.co/revistas/revistamba/Documents/ mba2011/6-redes-empresariales.pdf

Cox, S., Friesner, D. \& Khayun, M. (2003). Do reading skills courses help underprepared readers achieve academic success in college? Journal of College Reading and Learning, 33(2), 170-196. Doi: https://doi. org/10.1080/10790195.2003.10850147

Díaz, M., Pizza, N. y Salamanca, J. (2013). Proceso de internacionalización y aproximación a la logística de servicios: Telmex Colombia. Trabajo de grado. Universidad del Rosario. Bogotá, Colombia.

DHL, (2020). Estudio de DHL aportando resiliencia ante la pandemia como asegurar cadenas de suministro estables para vacunas y material sanitario durante la crisis del covid-19 y en emergencias sanitarias futuras. DHL. Recuperado en: https://www.dhl.com/content/dam/dhl/ global/core/documents/pdf/glo-core-delivering-pandemic-resilience-2020-Spanish.pdf

Dunning, J. (1981). International Production and the Multinational Enterprise. Londres, Inglaterra: Allen \& Urwin.

Dunning, J. (1988). The Eclectic Paradigm of International Production: A Restatement and some possible extensions. Journal of International Business Studies, 19(1), 1-31. Recuperado de https://www.jstor.org/ stable/154984?seq=1\#page_scan_tab_contents

Dunning, J. (1988a). Multinationals, Technology and Competitiveness. Londres, Inglaterra: Unwin Hyman.

Dunning, J. (1988b). Explaining International Production. Londres, Inglaterra: Unwin Hyman.

Dunning, J.H. (1992). Multinational Enterprises and the Global Economy. Londres, Inglaterra: Addison Welsey.

Ellis, P. (2000). Social ties and foreign market entry. Journal of international Business Studies, 31(3), 443-469. Recuperado de https://www. jstor.org/stable/155651?seq=1\#page_scan_tab_contents

Escobar, P. y Chaparro, S. (2011). Internacionalización exitosa de interconexión eléctrica ISA. Revista MBA EAFIT, 62-73. Recuperado de http://www.eafit.edu.co/revistas/revistamba/Documents/mba 2011/5-internacionalizacion-exitosa-isa.pdf 
Enfoka, (2020). El impacto de la covid-19 en la distribución de última milla. Enfoka-Trends septiembre 2020. Recuperado de: https://www. euskadi.eus/contenidos/documentacion/obs_mer_tendinnov_doc/ es_def/adjuntos/2020_09_tendencias_septiembre_2020.pdf

Federación Nacional de Cuero - Fedecuero. (2013). Federación Nacional de Cuero de Colombia. Plan de negocios del sector de cuero, calzado y marroquinería: una respuesta para la transformación productiva. Recuperado de https:/www.urosario.edu.co/urosario_files/portalurosario/7e/7e3381ed-ab23-4350-a971-8bbc6dc535bc.pdf

Ferrero, I. (2007). Milton Friedman (1912-2006). Libros de economia y empresa, (2), 64-67. Recuperado de http://www.librosdeeconomiayempresa.com/r004/articulo21.aspx

Ferrero, I. (2002). Milton Friedman: la politica económica de un pragmatista liberal. Madrid, España: Editorial Instituto de Estudios Económicos. Recuperado de http://dongdongnoodlebar.co.uk/ 2338189090/583518-8488533586.html

Fransoo, J., \& Udenio, M. (2020). Existing a Covid-19 lockdown: the bumpy road a head for many. Kuehne Logistics University. Research a center for operation Management.

Fraso, J. (2020). Estrategias para enfrentar la escalada de la demanda por COVID 19. Webinar realizado por el Logistic Sumi \& Expo.

Friedman, M. (1992). La Economía monetarista. Barcelona, España: Editorial Gedisa.

Freeman, R. (1984). Strategic Management: A Stakeholder Approach. Boston, EE. UU.: Pitman.

Ford, D. (1980). The development of buyer-seller relationships in industrial markets. European Journal of Marketing, 14(5/6).339-353. Doi: https://doi.org/10.1108/EUM0000000004910

Forrester, J. (1958). Industrial Dynamics: A Major Breakthrough for Decision Makers. Harvard Business Review, 36(4), 37-66. Recuperado de https:// kupdf.net/download/industrial-dynamics-a-major-breakthrough-for-decision-makers_5902479bdc0d603940959ed5_pdf

Forrester, J. (1961). Industrial Dynamics. Londres, Inglaterra: The M.I.T. Press.

Gómez, D. (2020). Sostenibilidad. Inclusión Y Desarrollo, 8(1), 131-143. Doi: https://doi.org/10.26620/uniminuto.inclusion.8.1.2021. 131-143 
Gómez, D., Carranza, Y. y Ramos, C. (2017a). Ecoturismo, turismo experiencial y cultural. Una reflexión con estudiantes de Administración de Turismo. Revisa temas, 3(11), 229-236. Recuperado de http://revistas.ustabuca.edu.co/index.php/TEMAS/article/view/ $1757 / 1347$

Gómez, D., Carranza, C. y Ramos, C. (2017b). Aportes de negociadores internacionales para el crecimiento, la innovación, el emprendimiento y la competitividad de las empresas colombianas Revista Facultad de Ciencias Contables, Económicas y Administrativas - FACCEA, 7(2), 157-164. Recuperado de http://www.udla.edu.co/revistas/index.php/ faccea/article/view/728/766

Gómez, D., Carranza, Y. y Ramos, C. (2016). Revisión documental, una herramienta para el mejoramiento de las competencias de lectura y escritura en estudiantes universitarios. Chakiñan, Revista de Ciencias Sociales y Humanidades, (1), 46-56. Recuperado de http://chakinan. unach.edu.ec/index.php/chakinan/article/view/10

Gómez, R. (2006). Logística Inversa un Proceso de Impacto Ambiental y Productivo. Revista Producción + Limpia, 5(2), 63-76. Recuperado de http://www.scielo.org.co/scielo.php?script=sci_arttext\&pid= 1909-04552010000200006\&lng=en\&nrm=iso\&tlng=es

González, J. y González, Ó. (2008). Determinantes de la proactividad medioambiental en la función logística. Cuadernos de estudios empresariales, 18. 51-72. Recuperado de https://dialnet.unirioja.es/ servlet/articulo? codigo $=3083642$

Gómez, E. y González, G. (2011). Proceso de internacionalización de empresas del área metropolitana de Bucaramanga. Lebret, (3), 57-81. Doi: https://doi.org/10.15332/rl.v0i3.46

Hayeck, F. (2008). Camino de servidumbre. Madrid, España: Editorial, S.A Harrington, H. (1996). Administración total del mejoramiento continuo. Bogotá, Colombia: McGraw Hill.

Harrington, H. (1998). Mejoramiento de los procesos de la empresa. Bogotá, Colombia: McGraw Hill.

Harrington, H. (1999). El proceso de mejoramiento. Milwaukee, EE.UU. Quality Press. 
Hymer, S. (1960). The international operations of national firms: A study of direct foreign investment (thesis doctoral). MIT. Cambridge, Inglaterra.

Kalmanovitz, S. (2019). Nueva historia económica de Colombia. Editorial Penguin Random House, Bogotá: Colombia.

Kojima. (1982). Macroeconomic versus international business approach to foreign direct investment. Hitotsubashi JournalofEconomics, 2(1), 1-19. Recuperado de https://www.jstor.org/stable/43295733?seq=1\#page_ scan_tab_contents

Larraya, J. (1994). La internacionalización de la economía y de las empresas vascas. Revista Vasca de economía, (30), 256-277. Recuperado de https://dialnet.unirioja.es/servlet/articulo?codigo=274324

Leporati, M., Morales, M., \& Martul, L. (2020). Las cadenas de suministro en la próxima pandemia. EAE Busines School. julio 2020. Recuperado de http://marketing.eae.es/prensa/SRC_CadenaSuministro.pdf?utm_source=Website\&utm_medium=Post_blog\&utm_ content=informe_cadenasuministro

Liendo, M., \& Martínez, A. (2011). Asociatividad. Una alternativa para el desarrollo y Crecimiento de las Pymes. Instituto de Investigaciones Económicas (Escuela de Economía No. 11). Recuperado de https:// www.fcecon.unr.edu.ar/web/sites/default/files/u16/Decimocuartas/ Liendo,\%20Martinez_asociatividad.pdf

Linton, T., \& Vakil, B. (2020). Coronavirus is Proving We Need More Resilient Supply Chains. Harvard Business Review.

Long, D. (2005). Logística Internacional: Administración de la cadena de abastecimiento global. México, DF, México: Lemus.

Organización para la Cooperación y el Desarrollo Económico - OECD. (2012). Evaluaciones de politicas nacionales de Educación. La Educación superior en Colombia. Recuperado de https://www.oecd.org/ education/skills-beyond-school/Evaluaciones $\% 20 \mathrm{de} \% 20 \mathrm{pol} \%$ C3\%ADticas\%20nacionales\%20de\%20Educaci\%C3\%B3n\%20 - \% 20 La \% 20 Educaci \% C3\% B 3n \% 20superior \% 20 en $\% 20$ Colombia.pdf 
Organización para la Cooperación y el Desarrollo Económico - OCDE. (2014). Panorama de la Educación Nota País. Recuperado de https:// www.oecd.org/edu/Colombia_EAG2014_CountryNote_ESP.pdf

Osorio, D. (2010). ¿ ¿Internacionalización con o son Desarrollo Local?: Un Asunto de Estrategia. Suma de Negocios, 1(1), 73 - 89. Recuperado de https://editorial.konradlorenz.edu.co/2010/12/internacionalizaci\%C3\%B3n-con-o-sin-desarrollo-local-un-asunto-de-estrategia.html

Peña, C., Torres, P., Vidal, C. y Marrnolejo, L. (2013). La logística de reversa y su relación con la gestión integral y sostenible de residuos sólidos en sectores productivos. Entramado, 9(1), 226-238. Doi: https://doi.org/10.18041/1900-3803/entramado.1.3455

Porter, M. (1985). Competitive Advantage: Creating and Sustaining Superior Performance. New York, EE. UU.: The free Press.

Rivera, L. (2020). Best practice Supply Chain COVID 19. Webinar realizado por la MIT CTL.

Reina, M. (2010). Internacionalización de la economía colombiana: comercio e inversión. Debates presidenciales CAF. Bogotá, Colombia: Fedesarrollo.

Rendón, J., y Gómez, D. (2020). Circuitos cortos y verdes. Alimentos si hay. Revista Sur. Recuperado en: https://www.sur.org.co/circuitos-cortos-y-verdes-alimentos-si-hay/

Root, F. (1994). Entry Strategies International Markets. Nueva York, EE. UU: Lexington Book.

Rueda, J. (2008). Los procesos de internacionalización de la empresa: causas y estrategias que lo promueven. Cuadernos Latinoamericanos de administración, 4, 751-757. Recuperado de https://masd.unbosque. edu.co/index.php/cuaderlam/article/view/1520/1126

Russo, M. \& Fouts, P. (1997). A Resource-Based Perspective on Corporate Environmental Performance and Profitability. The Academy of Management Journal, 40(3), 534-559. Doi: https://doi.org/10. $5465 / 257052$

Sáez, M. y Melo, C. (2002). La democracia brasileña, balance y perspectivas para el siglo XXI. Salamanca, Espańa: Editorial. Universidad de Salamanca. 
Salcedo, J. (2015). El docente universitario y el Sistema de comunicación de gubernamentalidad neoliberal. Revista Hojas y Hablas, (12), 20-40. Recuperado de http://revistas.unimonserrate.edu.co:8080/ hojasyhablas/article/view/2

Sagè-Gavin, E., Spitzer, B., Carrion, G., Feliciano, P., Good, T., \& Silverstone, Y. (2020). Human resilience: What your people need during COVID 19. Now text. Accenture.

Stiglitz, J. (2012). El precio de la desigualdad. Taurus. Barcelona: España. Stiglitz, J. (2020). Capitalismo progresista. La respuesta a la era del malestar. Taurus, Barcelona: España.

Tamayo, U., Azucena, M. y Molina, V. (2007). Generación de valor mediante prácticas de producción limpia, ecodiseño y logística inversa. Mediterráneo económico, (11), 147-164. Recuperado de https://dialnet.unirioja.es/servlet/articulo? codigo=2286879

Transport Intelliegence (2020). Global freight forwarding market sizing 2020: COVID19 impact analisis. Transport Intelligence Limited.

Urrea, L. y Abello, W. (2011). Análisis sobre la internacionalización de las Pymes en Colombia (Trabajo de grado). Universidad de la Sabana. Universidad de las Sabana. Bogotá, Colombia. Recuperado de https://intellectum.unisabana.edu.co/handle/10818/1857

Villarreal, O. (2005). La internacionalización de la empresa y la empresa multinacional: una revisión conceptual contemporánea. Cuadernos de Gestión, 5(2), 55 - 73. Recuperado de http://www.ehu.eus/ cuadernosdegestion/documentos/524.pdf

Villarreal, F. (2012). Logística Integral: una alternativa para crear valor y ventajas competitivas en las pequeñas y medianas empresas (pymes) del Sector Calzado. Nova scientia, 4(8), 165-202. Recuperado de http://www.scielo.org.mx/scielo.php?script=sci_arttext\&pid=S2007-07052012000200010

Velasco, N., y Gómez, D. (2020). La importancia de la Seguridady Salud en el Trabajo-SST para mejorar el clima organizacional. Segunda edición del Boletín de la Facultad de Ciencias Económicas y Administrativas de la Uniagustiniana Recuperado de: https:/www. uniagustiniana.edu.co/noticias/boletin-informativo-ndeg2-facultad-de-ciencias-economicas-y-administrativas 
Vernon, R. (1966). International investment and international trade in the product cycle. The Quarterly Journal of Economics, 80(2), 190-207. Doi: https://doi.org/10.2307/1880689

Wilding, R., Dohrmann, K., \& Wheatley, M. (2020). Reactivación de la cadena de suministro después del coronavirus. El viaje a la nueva normalidad. DHL. Recuperado en: https://simplydhl.com/Global/ FileLib/Spain/ES_DHL_PandemicWhitePaper_200828a.pdf

Zandi, M. (2020). Coronavirus: The Global Economic Threat. Moody's Analytics. 